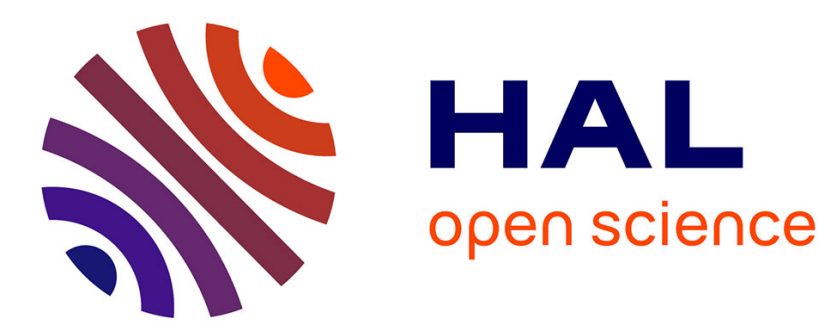

\title{
How good is my GAN?
}

Konstantin Shmelkov, Cordelia Schmid, Karteek Alahari

\section{To cite this version:}

Konstantin Shmelkov, Cordelia Schmid, Karteek Alahari. How good is my GAN?. ECCV 2018 European Conference on Computer Vision, Sep 2018, Munich, Germany. pp.218-234, 10.1007/978-3030-01216-8_14. hal-01850447

\section{HAL Id: hal-01850447 https://hal.inria.fr/hal-01850447}

Submitted on 27 Jul 2018

HAL is a multi-disciplinary open access archive for the deposit and dissemination of scientific research documents, whether they are published or not. The documents may come from teaching and research institutions in France or abroad, or from public or private research centers.
L'archive ouverte pluridisciplinaire HAL, est destinée au dépôt et à la diffusion de documents scientifiques de niveau recherche, publiés ou non, émanant des établissements d'enseignement et de recherche français ou étrangers, des laboratoires publics ou privés. 


\title{
How good is my GAN?
}

\author{
Konstantin Shmelkov, Cordelia Schmid, and Karteek Alahari \\ $\operatorname{Inria}^{\star}$
}

\begin{abstract}
Generative adversarial networks (GANs) are one of the most popular methods for generating images today. While impressive results have been validated by visual inspection, a number of quantitative criteria have emerged only recently. We argue here that the existing ones are insufficient and need to be in adequation with the task at hand. In this paper we introduce two measures based on image classification-GANtrain and GAN-test, which approximate the recall (diversity) and precision (quality of the image) of GANs respectively. We evaluate a number of recent GAN approaches based on these two measures and demonstrate a clear difference in performance. Furthermore, we observe that the increasing difficulty of the dataset, from CIFAR10 over CIFAR100 to ImageNet, shows an inverse correlation with the quality of the GANs, as clearly evident from our measures.
\end{abstract}

\section{Introduction}

Generative Adversarial Networks (GANs) [19] are deep neural net architectures composed of a pair of competing neural networks: a generator and a discriminator. This model is trained by alternately optimizing two objective functions so that the generator $G$ learns to produce samples resembling real images, and the discriminator $D$ learns to better discriminate between real and fake data. Such a paradigm has huge potential, as it can learn to generate any data distribution. This has been exploited with some success in several computer vision problems, such as text-to-image [56] and image-to-image [24,59] translation, super-resolution [31], and realistic natural image generation [25].

Since the original GAN model [19] was proposed, many variants have appeared in the past few years, for example, to improve the quality of the generated images $[12,15,25,36]$, or to stabilize the training procedure $[7,9,20,34,36,40,57]$. GANs have also been modified to generate images of a given class by conditioning on additional information, such as the class label $[16,35,37,41]$. There are a number of ways to do this: ranging from concatenation of label $y$ to the generator input $\mathbf{z}$ or intermediate feature maps [16,35], to using conditional batch normalization [37], and augmenting the discriminator with an auxiliary classifier [41].

\footnotetext{
* Univ. Grenoble Alpes, Inria, CNRS, Grenoble INP, LJK, 38000 Grenoble, France. This work was supported in part by the ERC advanced grant ALLEGRO, gifts from Amazon, Facebook and Intel, and the Indo-French project EVEREST (no. 5302-1) funded by CEFIPRA.
} 


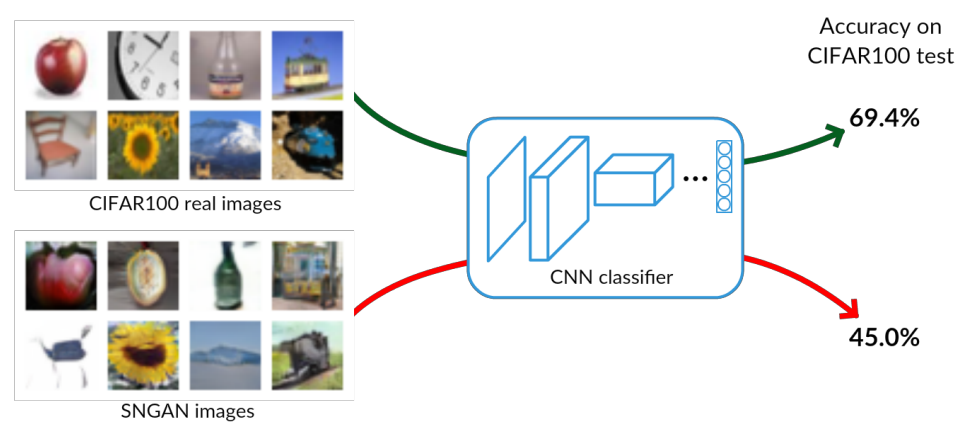

Fig. 1: State-of-the-art GANs, e.g., SNGAN [36], generate realistic images, which are difficult to evaluate subjectively in comparison to real images. Our new image classification accuracy-based measure (GAN-train is shown here) overcomes this issue, showing a clear difference between real and generated images.

With several such variants being regularly proposed in the literature, a critical question is how these models can be evaluated and compared to each other.

Evaluation and comparison of GANs, or equivalently, the images generated by GANs, is challenging. This is in part due to the lack of an explicit likelihood measure [51], which is commonplace in comparable probabilistic models [27, 47]. Thus, much of the previous work has resorted to a mere subjective visual evaluation in the case of images synthesized by GANs. As seen from the sample images generated by a state-of-the-art GAN [36] in Figure 1, it is impossible to judge their quality precisely with a subjective evaluation. Recent work in the past two years has begun to target this challenge through quantitative measures for evaluating GANs $[22,25,32,46]$.

Inception score (IS) [46] and Fréchet Inception distance (FID) [22] were suggested as ad-hoc measures correlated with the visual quality of generated images. Inception score measures the quality of a generated image by computing the KLdivergence between the (logit) response produced by this image and the marginal distribution, i.e., the average response of all the generated images, using an Inception network [50] trained on ImageNet. In other words, Inception score does not compare samples with a target distribution, and is limited to quantifying the diversity of generated samples. Fréchet Inception distance compares Inception activations (responses of the penultimate layer of the Inception network) between real and generated images. This comparison however approximates the activations of real and generated images as Gaussian distributions (cf. equation (2)), computing their means and covariances, which are too crude to capture subtle details. Both these measures rely on an ImageNet-pretrained Inception network, which is far from ideal for other datasets, such as faces and biomedical imaging. Overall, IS and FID are useful measures to evaluate how training advances, but they guarantee no correlation with performance on real-world tasks. As we discuss in Section 5, these measures are insufficient to finely separate 
state-of-the-art GAN models, unlike our measures (see SNGAN vs WPGAN-GP (10M) in Table 2 for example).

An alternative evaluation is to compute the distance of the generated samples to the real data manifold in terms of precision and recall [32]. Here, high precision implies that the generated samples are close to the data manifold, and high recall shows that the generator outputs samples that cover the manifold well. These measures remain idealistic as they are impossible to compute on natural image data, whose manifold is unknown. Indeed, the evaluation in [32] is limited to using synthetic data composed of gray-scale triangles. Another distance suggested for comparing GAN models is sliced Wasserstein distance (SWD) [25]. SWD is an approximation of Wasserstein-1 distance between real and generated images, and is computed as the statistical similarity between local image patches extracted from Laplacian pyramid representations of these images. As shown in Section 5, SWD is less-informative than our evaluation measures.

In this paper, we propose new evaluation measures to compare class-conditional GAN architectures with GAN-train and GAN-test scores. We rely on a neural net architecture for image classification for both these measures. To compute GAN-train, we train a classification network with images generated by a GAN, and then evaluate its performance on a test set composed of real-world images. Intuitively, this measures the difference between the learned (i.e., generated image) and the target (i.e., real image) distributions. We can conclude that generated images are similar to real ones if the classification network, which learns features for discriminating images generated for different classes, can correctly classify real images. In other words, GAN-train is akin to a recall measure, as a good GAN-train performance shows that the generated samples are diverse enough. However, GAN-train also requires a sufficient precision, as otherwise the classifier will be impacted by the sample quality.

Our second measure, GAN-test, is the accuracy of a network trained on real images and evaluated on the generated images. This measure is similar to precision, with a high value denoting that the generated samples are a realistic approximation of the (unknown) distribution of natural images. In addition to these two measures, we study the utility of images generated by GANs for augmenting training data. This can be interpreted as a measure of the diversity of the generated images. The utility of our evaluation approach, in particular, when a subjective inspection is insufficient, is illustrated with the GAN-train measure in Figure 1. We will discuss these measures in detail in Section 3.

As shown in our extensive experimental results in Section 5 and the appendix in the supplementary material and technical report [5], these measures are much more informative to evaluate GANs, compared to all the previous measures discussed, including cases where human studies are inconclusive. In particular, we evaluate two state-of-the-art GAN models: WGAN-GP [20] and SNGAN [36], along with other generative models [45, 47] to provide baseline comparisons. Image classification performance is evaluated on MNIST [30], CIFAR10, CIFAR100 [28], and the ImageNet [14] datasets. Experimental results 
show that the quality of GAN images decreases significantly as the complexity of the dataset increases.

\section{Related work}

We present existing quantitative measures to evaluate GANs: scores based on an Inception network, i.e., IS and FID, a Wasserstein-based distance metric, precision and recall scores, and a technique built with data augmentation.

\subsection{Inception score}

One of the most common ways to evaluate GANs is the Inception score [46]. It uses an Inception network [50] pre-trained on ImageNet to compute logits of generated images. The score is given by:

$$
\operatorname{IS}(G)=\exp \left(\underset{\mathbf{x} \sim p_{g}}{\mathbb{E}}\left[D_{\mathrm{KL}}(p(y \mid \mathbf{x}) \| p(y))\right]\right),
$$

where $\mathbf{x}$ is a generated image sampled from the learned generator distribution $p_{g}$, $\mathbb{E}$ is the expectation over the set of generated images, $D_{\mathrm{KL}}$ is the KL-divergence between the conditional class distribution $p(y \mid \mathbf{x})$ (for label $y$, according to the Inception network) and the marginal class distribution $p(y)=\underset{\mathbf{x} \sim p_{g}}{\mathbb{E}}[p(y \mid \mathbf{x})]$. By definition, Inception score does not consider real images at all, and so cannot measure how well the generator approximates the real distribution. This score is limited to measuring only the diversity of generated images. Some of its other limitations, as noted in [8], are: high sensitivity to small changes in weights of the Inception network, and large variance of scores.

\subsection{Fréchet Inception distance}

The recently proposed Fréchet Inception distance (FID) [22] compares the distributions of Inception embeddings (activations from the penultimate layer of the Inception network) of real $\left(p_{r}(\mathbf{x})\right)$ and generated $\left(p_{g}(\mathbf{x})\right)$ images. Both these distributions as modeled as multi-dimensional Gaussians parameterized by their respective mean and covariance. The distance measure is defined between the two Gaussian distributions as:

$$
d^{2}\left(\left(\mathbf{m}_{r}, \mathbf{C}_{r}\right),\left(\mathbf{m}_{g}, \mathbf{C}_{g}\right)\right)=\left\|\mathbf{m}_{r}-\mathbf{m}_{g}\right\|^{2}+\operatorname{Tr}\left(\mathbf{C}_{r}+\mathbf{C}_{g}-2\left(\mathbf{C}_{r} \mathbf{C}_{g}\right)^{\frac{1}{2}}\right),
$$

where $\left(\mathbf{m}_{r}, \mathbf{C}_{r}\right),\left(\mathbf{m}_{g}, \mathbf{C}_{g}\right)$ denote the mean and covariance of the real and generated image distributions respectively. FID is inversely correlated with Inception score, and suffers from the same issues discussed earlier.

The two Inception-based measures cannot separate image quality from image diversity. For example, low IS or FID values can be due to the generated images being either not realistic (low image quality) or too similar to each other (low diversity), with no way to analyze the cause. In contrast, our measures can distinguish when generated images become less diverse from worse image quality. 


\subsection{Other evaluation measures}

Sliced Wasserstein distance (SWD) [25] was used to evaluate high-resolution GANs. It is a multi-scale statistical similarity computed on local image patches extracted from the Laplacian pyramid representation of real and generated images. A total of $1287 \times 7$ local patches for each level of the Laplacian pyramid are extracted per image. While SWD is an efficient approximation, using randomized projections [44], of the Wasserstein-1 distance between the real and generated images, its utility is limited when comparing a variety of GAN models, with not all of them producing high-resolution images (see our evaluation in Section 5).

Precision and recall measures were introduced [32] in the context of GANs, by constructing a synthetic data manifold. This makes it possible to compute the distance of an image sample (generated or real) to the manifold, by finding its distance to the closest point from the manifold. In this synthetic setup, precision is defined as the fraction of the generated samples whose distance to the manifold is below a certain threshold. Recall, on the other hand, is computed by considering a set of test samples. First, the latent representation $\tilde{\mathbf{z}}$ of each test sample $\mathbf{x}$ is estimated, through gradient descent, by inverting the generator $G$. Recall is then given by the fraction of test samples whose L2-distance to $G(\tilde{\mathbf{z}})$ is below the threshold. High recall is equivalent to the GAN capturing most of the manifold, and high precision implies that the generated samples are close to the manifold. Although these measures bring the flavor of techniques used widely to evaluate discriminative models to GANs, they are impractical for real images as the data manifold is unknown, and their use is limited to evaluations on synthetic data [32].

\subsection{Data augmentation}

Augmenting training data is an important component of learning neural networks. This can be achieved by increasing the size of the training set [29] or incorporating augmentation directly in the latent space [54]. A popular technique is to increase the size of the training set with minor transformations of data, which has resulted in a performance boost, e.g., for image classification [29]. GANs provide a natural way to augment training data with the generated samples. Indeed, GANs have been used to train classification networks in a semi-supervised fashion [13,52] or to facilitate domain adaptation [10]. Modern GANs generate images realistic enough to improve performance in applications, such as, biomedical imaging $[11,18]$, person re-identification [58] and image enhancement [55]. They can also be used to refine training sets composed of synthetic images for applications such as eye gaze and hand pose estimation [49]. GANs are also used to learn complex 3D distributions and replace computationally intensive simulations in physics [39,42] and neuroscience [38]. Ideally, GANs should be able to recreate the training set with different variations. This can be used to compress datasets for learning incrementally, without suffering from catastrophic forgetting as new classes are added [48]. We will study the utility of GANs for 


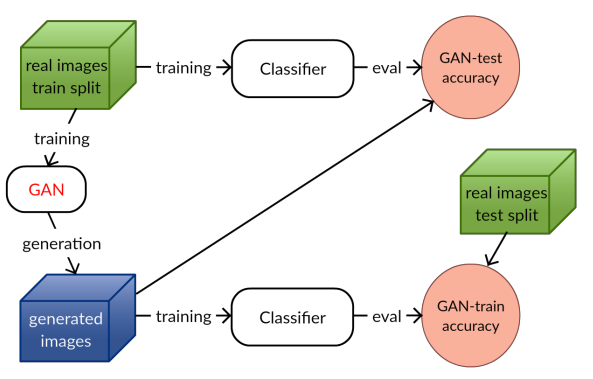

Fig. 2: Illustration of GAN-train and GAN-test. GAN-train learns a classifier on GAN generated images and measures the performance on real test images. This evaluates the diversity and realism of GAN images. GAN-test learns a classifier on real images and evaluates it on GAN images. This measures how realistic GAN images are.

training image classification networks with data augmentation (see Section 5.4), and analyze it as an evaluation measure.

In summary, evaluation of generative models is not a easy task [51], especially for models like GANs. We bring a new dimension to this problem with our GAN-train and GAN-test performance-based measures, and show through our extensive analysis that they are complementary to all the above schemes.

\section{GAN-train and GAN-test}

An important characteristic of a conditional GAN model is that generated images should not only be realistic, but also recognizable as coming from a given class. An optimal GAN that perfectly captures the target distribution can generate a new set of images $S_{g}$, which are indistinguishable from the original training set $S_{t}$. Assuming both these sets have the same size, a classifier trained on either of them should produce roughly the same validation accuracy. This is indeed true when the dataset is simple enough, for example, MNIST [48] (see also Section 5.2). Motivated by this optimal GAN characteristic, we devise two scores to evaluate GANs, as illustrated in Figure 2.

GAN-train is the accuracy of a classifier trained on $S_{g}$ and tested on a validation set of real images $S_{v}$. When a GAN is not perfect, GAN-train accuracy will be lower than the typical validation accuracy of the classifier trained on $S_{t}$. It can happen due to many reasons, e.g., (i) mode dropping reduces the diversity of $S_{g}$ in comparison to $S_{t}$, (ii) generated samples are not realistic enough to make the classifier learn relevant features, (iii) GANs can mix-up classes and confuse the classifier. Unfortunately, GAN failures are difficult to diagnose. When GANtrain accuracy is close to validation accuracy, it means that GAN images are high quality and as diverse as the training set. As we will show in Section 5.3, diversity varies with the number of generated images. We will analyze this with the evaluation discussed at the end of this section.

GAN-test is the accuracy of a classifier trained on the original training set $S_{t}$, but tested on $S_{g}$. If a GAN learns well, this turns out be an easy task because both the sets have the same distribution. Ideally, GAN-test should be close to the validation accuracy. If it significantly higher, it means that the GAN overfits, and simply memorizes the training set. On the contrary, if it is significantly lower, 
the GAN does not capture the target distribution well and the image quality is poor. Note that this measure does not capture the diversity of samples because a model that memorizes exactly one training image perfectly will score very well. GAN-test accuracy is related to the precision score in [32], quantifying how close generated images are to a data manifold.

To provide an insight into the diversity of GAN-generated images, we measure GAN-train accuracy with generated sets of different sizes, and compare it with the validation accuracy of a classifier trained on real data of the corresponding size. If all the generated images were perfect, the size of $S_{g}$ where GAN-train is equal to validation accuracy with the reduced-size training set, would be a good estimation of the number of distinct images in $S_{g}$. In practice, we observe that GAN-train accuracy saturates with a certain number of GAN-generated samples (see Figures 4(a) and 4(b) discussed in Section 5.3). This is a measure of the diversity of a GAN, similar to recall from [32], measuring the fraction of the data manifold covered by a GAN.

\section{Datasets and Methods}

Datasets. For comparing the different GAN methods and PixelCNN++, we use several image classification datasets with an increasing number of labels: MNIST [30], CIFAR10 [28], CIFAR100 [28] and ImageNet1k [14]. CIFAR10 and CIFAR100 both have $50 \mathrm{k} 32 \times 32$ RGB images in the training set, and 10k images in the validation set. CIFAR10 has 10 classes while CIFAR100 has 100 classes. ImageNet1k has 1000 classes with $1.3 \mathrm{M}$ training and 50k validation images. We downsample the original ImageNet images to two resolutions in our experiments, namely $64 \times 64$ and $128 \times 128$. MNIST has 10 classes of $28 \times 28$ grayscale images, with $60 \mathrm{k}$ samples for training and $10 \mathrm{k}$ for validation.

We exclude the CIFAR10/CIFAR100/ImageNet1k validation images from GAN training to enable the evaluation of test accuracy. This is not done in a number of GAN papers and may explain minor differences in IS and FID scores compared to the ones reported in these papers.

\subsection{Evaluated methods}

Among the plethora of GAN models in literature, it is difficult to choose the best one, especially since appropriate hyperparameter fine-tuning appears to bring all major GANs within a very close performance range, as noted in a study [32]. We choose to perform our analysis on Wasserstein GAN (WGAN-GP), one of the most widely-accepted models in literature at the moment, and SNGAN, a very recent model showing state-of-the-art image generation results on ImageNet. Additionally, we include two baseline generative models, DCGAN [45] and PixelCNN $++[47]$. We summarize all the models included in our experimental analysis below, and present implementation details in the appendix [5].

Wasserstein GAN. WGAN [7] replaces the discriminator separating real and generated images with a critic estimating Wasserstein-1 (i.e., earth-mover's) dis- 
tance between their corresponding distributions. The success of WGANs in comparison to the classical GAN model [19] can be attributed to two reasons. Firstly, the optimization of the generator is easier because the gradient of the critic function is better behaved than its GAN equivalent. Secondly, empirical observations show that the WGAN value function better correlates with the quality of the samples than GANs [7].

In order to estimate the Wasserstein-1 distance between the real and generated image distributions, the critic must be a K-Lipschitz function. The original paper [7] proposed to constrain the critic through weight clipping to satisfy this Lipschitz requirement. This, however, can lead to unstable training or generate poor samples [20]. An alternative to clipping weights is the use of a gradient penalty as a regularizer to enforce the Lipschitz constraint. In particular, we penalize the norm of the gradient of the critic function with respect to its input. This has demonstrated stable training of several GAN architectures [20].

We use the gradient penalty variant of WGAN, conditioned on data in our experiments, and refer to it as WGAN-GP in the rest of the paper. Label conditioning is an effective way to use labels available in image classification training data [41]. Following ACGAN [41], we concatenate the noise input $\mathbf{z}$ with the class label in the generator, and modify the discriminator to produce probability distributions over the sources as well as the labels.

SNGAN. Variants have also analyzed other issues related to training GANs, such as the impact of the performance control of the discriminator on training the generator. Generators often fail to learn the multimodal structure of the target distribution due to unstable training of the discriminator, particularly in highdimensional spaces [36]. More dramatically, generators cease to learn when the supports of the real and the generated image distributions are disjoint [6]. This occurs since the discriminator quickly learns to distinguish these distributions, resulting in the gradients of the discriminator function, with respect to the input, becoming zeros, and thus failing to update the generator model any further.

SNGAN [36] introduces spectral normalization to stabilize training the discriminator. This is achieved by normalizing each layer of the discriminator (i.e., the learnt weights) with the spectral norm of the weight matrix, which is its largest singular value. Miyato et al. [36] showed that this regularization outperforms other alternatives, including gradient penalty, and in particular, achieves state-of-the-art image synthesis results on ImageNet. We use the classconditioned version of SNGAN [37] in our evaluation. Here, SNGAN is conditioned with projection in the discriminator network, and conditional batch normalization [17] in the generator network.

DCGAN. Deep convolutional GANs (DCGANs) is a class of architecture that was proposed to leverage the benefits of supervised learning with CNNs as well as the unsupervised learning of GAN models [45]. The main principles behind DCGANs are using only convolutional layers and batch normalization for the generator and discriminator networks. Several instantiations of DCGAN are possible with these broad guidelines, and in fact, many do exist in literature [20,36, 41]. We use the class-conditioned variant presented in [41] for our analysis. 
PixelCNN++. The original PixelCNN [53] belongs to a class of generative models with tractable likelihood. It is a deep neural net which predicts pixels sequentially along both the spatial dimensions. The spatial dependencies among pixels are captured with a fully convolutional network using masked convolutions. PixelCNN++ proposes improvements to this model in terms of regularization, modified network connections and more efficient training [47].

\section{$5 \quad$ Experiments}

\subsection{Implementation details of evaluation measures}

We compute Inception score with the WGAN-GP code [1] corrected for the 1008 classes problem [8]. The mean value of this score computed 10 times on $5 \mathrm{k}$ splits is reported in all our evaluations, following standard protocol.

We found that there are two variants for computing FID. The first one is the original implementation [2] from the authors [22], where all the real images and at least 10k generated images are used. The second one is from the SNGAN [36] implementation, where $5 \mathrm{k}$ generated images are compared to $5 \mathrm{k}$ real images. Estimation of the covariance matrix is also different in both these cases. Hence, we include these two versions of FID in the paper to facilitate comparison in the future. The original implementation is referred to as FID, while our implementation [4] of the $5 \mathrm{k}$ version is denoted as FID-5K. Implementation of SWD is taken from the official NVIDIA repository [3].

\subsection{Generative model evaluation}

MNIST. We validate our claim (from Section 3) that a GAN can perfectly reproduce a simple dataset on MNIST. A four-layer convnet classifier trained on real MNIST data achieves $99.3 \%$ accuracy on the test set. In contrast, images generated with SNGAN achieve a GAN-train accuracy of $99.0 \%$ and GAN-test accuracy of $99.2 \%$, highlighting their high image quality as well as diversity.

CIFAR10. Table 1 shows a comparison of state-of-the-art GAN models on CIFAR10. We observe that the relative ranking of models is consistent across different metrics: FID, GAN-train and GAN-test accuracies. Both GAN-train and GAN-test are quite high for SNGAN and WGAN-GP (10M). This implies that both the image quality and the diversity are good, but are still lower than that of real images (92.8 in the first row). Note that PixelCNN++ has low diversity because GAN-test is much higher than GAN-train in this case. This is in line with its relatively poor Inception score and FID (as shown in [32] FID is quite sensitive to mode dropping).

Note that SWD does not correlate well with other metrics: it is consistently smaller for WGAN-GP (especially SWD 32). We hypothesize that this is because SWD approximates the Wasserstein-1 distance between patches of real and generated images, which is related to the optimization objective of Wasserstein GANs, but not other models (e.g., SNGAN). This suggests that SWD is 


\begin{tabular}{l|r|r|r|r|r|r|r} 
model & IS & FID-5K & FID & $\begin{array}{r}\text { GAN- } \\
\text { train }\end{array}$ & $\begin{array}{r}\text { GAN- } \\
\text { test }\end{array}$ & SWD 16 & SWD 32 \\
\hline real images & 11.33 & 9.4 & 2.1 & 92.8 & - & 2.8 & 2.0 \\
SNGAN & 8.43 & 18.8 & 11.8 & 82.2 & 87.3 & 3.9 & 24.4 \\
WGAN-GP (10M) & 8.21 & 21.5 & 14.1 & 79.5 & 85.0 & 3.8 & 6.2 \\
WGAN-GP (2.5M) & 8.29 & 22.1 & 15.0 & 76.1 & 80.7 & 3.4 & 6.9 \\
DCGAN & 6.69 & 42.5 & 35.6 & 65.0 & 58.2 & 6.5 & 24.7 \\
PixelCNN++ & 5.36 & 121.3 & 119.5 & 34.0 & 47.1 & 14.9 & 56.6
\end{tabular}

Table 1: CIFAR10 experiments. IS: higher is better. FID and SWD: lower is better. SWD values here are multiplied by $10^{3}$ for better readability. GAN-train and GAN-test are accuracies given as percentage (higher is better).

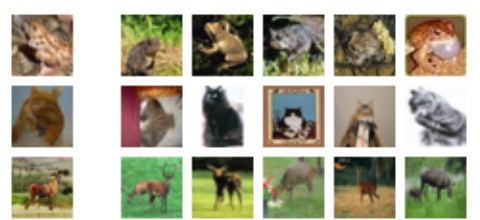

Fig. 3: First column: SNGAN-generated images. Other columns: 5 images from CIFAR10 "train" closest to GAN image from the first column in feature space of baseline CIFAR10 classifier.

unsuitable to compare WGAN and other GAN losses. It is also worth noting that WGAN-GP (10M) shows only a small improvement over WGAN-GP (2.5M) despite a four-fold increase in the number of parameters. In Figure 3 we show SNGAN-generated images on CIFAR10 and their nearest neighbors from the training set in the feature space of the classifier we use to compute the GANtest measure. Note that SNGAN consistently finds images of the same class as a generated image, which are close to an image from the training set.

To highlight the complementarity of GAN-train and GAN-test, we emulate a simple model by subsampling/corrupting the CIFAR10 training set, in the spirit of [22]. GAN-train/test now corresponds to training/testing the classifier on modified data. We observe that GAN-test is insensitive to subsampling unlike GAN-train (where it is equivalent to training a classifier on a smaller split). Salt and pepper noise, ranging from $1 \%$ to $20 \%$ of replaced pixels per image, barely affects GAN-train, but degrades GAN-test significantly (from $82 \%$ to 15\%).

Through this experiment on modified data, we also observe that FID is insufficient to distinguish between the impact of image diversity and quality. For example, FID between CIFAR10 train set and train set with Gaussian noise $(\sigma=5)$ is 27.1, while FID between train set and its random $5 \mathrm{k}$ subset with the same noise is 29.6. This difference may be due to lack of diversity or quality or both. GAN-test, which measures the quality of images, is identical (95\%) in both these cases. GAN-train, on the other hand, drops from $91 \%$ to $80 \%$, showing that the $5 \mathrm{k}$ train set lacks diversity. Together, our measures, address one of the main drawbacks of FID.

CIFAR100. Our results on CIFAR100 are summarized in Table 2. It is a more challenging dataset than CIFAR10, mainly due to the larger number of classes and fewer images per class; as evident from the accuracy of a convnet for clas- 
sification trained with real images: 92.8 vs 69.4 for CIFAR10 and CIFAR100 respectively. SNGAN and WGAN-GP (10M) produce similar IS and FID, but very different GAN-train and GAN-test accuracies. This makes it easier to conclude that SNGAN has better image quality and diversity than WGAN-GP (10M). It is also interesting to note that WGAN-GP (10M) is superior to WGAN-GP $(2.5 \mathrm{M})$ in all the metrics, except SWD. WGAN-GP $(2.5 \mathrm{M})$ achieves reasonable IS and FID, but the quality of the generated samples is very low, as evidenced by GAN-test accuracy. SWD follows the same pattern as in the CIFAR10 case: WGAN-GP shows a better performance than others in this measure, which is not consistent with its relatively poor image quality. PixelCNN ++ exhibits an interesting behavior, with high GAN-test accuracy, but very low GAN-train accuracy, showing that it can generate images of acceptable quality, but they lack diversity. A high FID in this case also hints at significant mode dropping. We also analyze the quality of the generated images with t-SNE [33] in the appendix [5].

Random forests. We verify if our findings depend on the type of classifier by using random forests $[23,43]$ instead of CNN for classification. This results in GANtrain, GAN-test scores of $15.2 \%, 19.5 \%$ for SNGAN, $10.9 \%, 16.6 \%$ for WGANGP $(10 \mathrm{M}), 3.7 \%, 4.8 \%$ for WGAN-GP $(2.5 \mathrm{M})$, and $3.2 \%, 3.0 \%$ for DCGAN respectively. Note that the relative ranking of these GANs remains identical for random forests and CNNs.

Human study. We designed a human study with the goal of finding which of the measures (if any) is better aligned with human judgement. The subjects were asked to choose the more realistic image from two samples generated for a particular class of CIFAR100. Five subjects evaluated SNGAN vs one of the following: DCGAN, WGAN-GP (2.5M), WGAN-GP (10M) in three separate tests. They made 100 comparisons of randomly generated image pairs for each test, i.e., 1500 trials in total. All of them found the task challenging, in particular for both WGAN-GP tests.

We use Student's t-test for statistical analysis of these results. In SNGAN vs DCGAN, subjects chose SNGAN 368 out of 500 trials, in SNGAN vs WGANGP (2.5M), subjects preferred SNGAN 274 out of 500 trials, and in SNGAN vs WGAN-GP (10M), SNGAN was preferred 230 out of 500 . The preference of SNGAN over DCGAN is statistically significant $\left(p<10^{-7}\right)$, while the preference over WGAN-GP $(2.5 \mathrm{M})$ or WGAN-GP $(10 \mathrm{M})$ is insignificant $(p=0.28$ and $p=0.37$ correspondingly). We conclude that the quality of images generated needs to be significantly different, as in the case of SNGAN vs DCGAN, for human studies to be conclusive. They are insufficient to pick out the subtle, but performance-critical, differences, unlike our measures.

ImageNet. On this dataset, which is one of the more challenging ones for image synthesis [36], we analyzed the performance of the two best GAN models based on our CIFAR experiments, i.e., SNGAN and WGAN-GP. As shown in Table 3, SNGAN achieves a reasonable GAN-train accuracy and a relatively high GANtest accuracy at $128 \times 128$ resolution. This suggests that SNGAN generated images have good quality, but their diversity is much lower than the original data. This may be partly due to the size of the generator (150Mb) being significantly 


\begin{tabular}{l|r|r|r|r|r|r|r} 
model & IS & FID-5K & FID & $\begin{array}{r}\text { GAN- } \\
\text { train }\end{array}$ & $\begin{array}{r}\text { GAN- } \\
\text { test }\end{array}$ & SWD 16 & SWD 32 \\
\hline real images & 14.9 & 10.8 & 2.4 & 69.4 & - & 2.7 & 2.0 \\
SNGAN & 9.30 & 23.8 & 15.6 & 45.0 & 59.4 & 4.0 & 15.6 \\
WGAN-GP (10M) & 9.10 & 23.5 & 15.6 & 26.7 & 40.4 & 6.0 & 9.1 \\
WGAN-GP (2.5M) & 8.22 & 28.8 & 20.6 & 5.4 & 4.3 & 3.7 & 7.7 \\
DCGAN & 6.20 & 49.7 & 41.8 & 3.5 & 2.4 & 9.9 & 20.8 \\
PixelCNN++ & 6.27 & 143.4 & 141.9 & 4.8 & 27.5 & 8.5 & 25.9
\end{tabular}

Table 2: CIFAR100 experiments. Refer to the caption of Table 1 for details.

\begin{tabular}{|c|c|c|c|c|c|c|c|c|}
\hline res & model & IS & FID-5K & FID & $\begin{array}{r}\text { GAN- } \\
\text { train } \\
\text { top-1 }\end{array}$ & $\begin{array}{r}\text { GAN- } \\
\text { train } \\
\text { top-5 }\end{array}$ & $\begin{array}{r}\text { GAN- } \\
\text { test } \\
\text { top- } 1\end{array}$ & $\begin{array}{r}\text { GAN- } \\
\text { test } \\
\text { top-5 }\end{array}$ \\
\hline \multirow{3}{*}{$64 \mathrm{px}$} & real images & 63.8 & 15.6 & 2.9 & 55.0 & 78.8 & - & - \\
\hline & SNGAN & 12.3 & 44.5 & 34.4 & 3 & 8.4 & 12.9 & 28.9 \\
\hline & WGAN-GP & 11.3 & 46.7 & 35.8 & 0.1 & 0.7 & 0.1 & 0.5 \\
\hline \multirow{3}{*}{$128 \mathrm{px}$} & real images & 203.2 & 17.4 & 3.0 & 59.1 & 81.9 & - & - \\
\hline & SNGAN* & 35.3 & 44.9 & 33.2 & 9.3 & 21.9 & 39.5 & 63.4 \\
\hline & WGAN-GP & 11.6 & 91.6 & 79.5 & 0.1 & 0.5 & 0.1 & 0.5 \\
\hline
\end{tabular}

Table 3: ImageNet experiments. SNGAN* refers to the model provided by [36], trained for $850 \mathrm{k}$ iterations. Refer to the caption of Table 1 for details.

smaller in comparison to ImageNet training data $(64 \mathrm{~Gb}$ for $128 \times 128)$. Despite this difference in size, it achieves GAN-train accuracy of $9.3 \%$ and $21.9 \%$ for top1 and top- 5 classification results respectively. In comparison, the performance of WGAN-GP is dramatically poorer; see last row for each resolution in the table.

In the case of images generated at $64 \times 64$ resolution, GAN-train and GANtest accuracies with SNGAN are lower than their $128 \times 128$ counterparts. GANtest accuracy is over four times better than GAN-train, showing that the generated images lack in diversity. It is interesting to note that WGAN-GP produces Inception score and FID very similar to SNGAN, but its images are insufficient to train a reasonable classifier and to be recognized by an ImageNet classifier, as shown by the very low GAN-train and GAN-test scores.

\subsection{GAN image diversity}

We further analyze the diversity of the generated images by evaluating GANtrain accuracy with varying amounts of generated data. A model with low diversity generates redundant samples, and increasing the quantity of data generated in this case does not result in better GAN-train accuracy. In contrast, generating more samples from a model with high diversity produces a better GAN-train score. We show this analysis in Figure 4, where GAN-train accuracy is plotted with respect to the size of the generated training set on CIFAR10 and CIFAR100.

In the case of CIFAR10, we observe that GAN-train accuracy saturates around 15-20k generated images, even for the best model SNGAN (see Fig- 

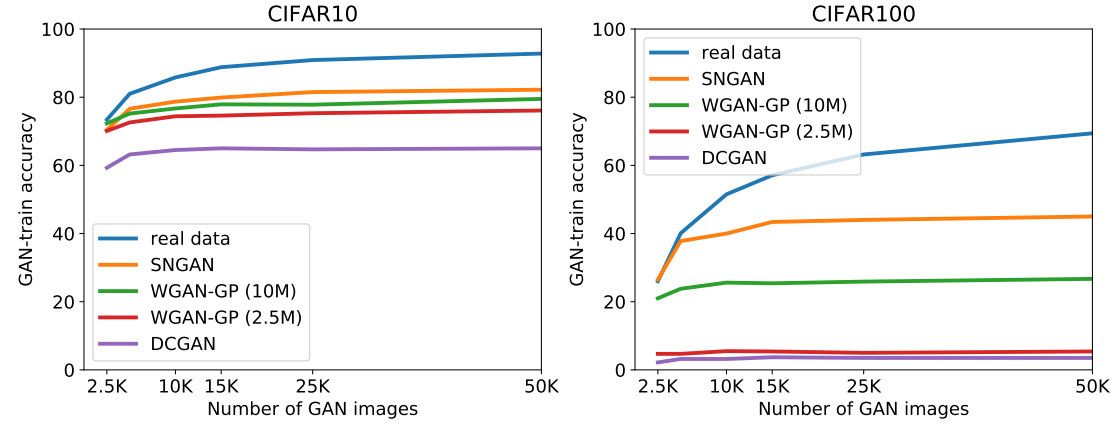

Fig. 4: The effect of varying the size of the generated image set on GAN-train accuracy. For comparison, we also show the result (in blue) of varying the size of the real image training dataset. (Best viewed in pdf.)
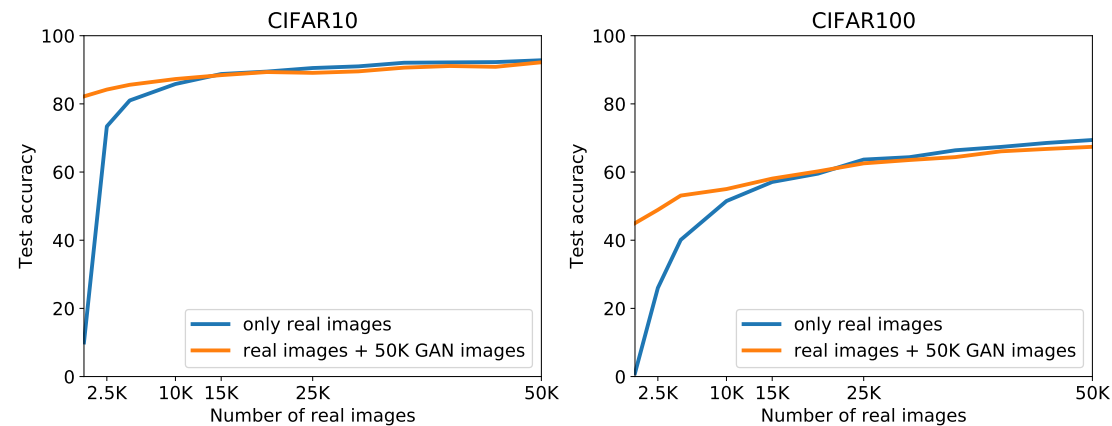

Fig. 5: The impact of training a classifier with a combination of real and SNGAN generated images.

ure 4a). With DCGAN, which is weaker than SNGAN, GAN-train saturates around $5 \mathrm{k}$ images, due to its relatively poorer diversity. Figure $4 \mathrm{~b}$ shows no increase in GAN-train accuracy on CIFAR100 beyond 25k images for all the models. The diversity of $5 \mathrm{k}$ SNGAN-generated images is comparable to the same quantity of real images; see blue and orange plots in Figure 4b. WGAN-GP (10M) has very low diversity beyond $5 \mathrm{k}$ generated images. WGAN-GP $(2.5 \mathrm{M})$ and DCGAN perform poorly on CIFAR100, and are not competitive with respect to the other methods.

\subsection{GAN data augmentation}

We analyze the utility of GANs for data augmentation, i.e., for generating additional training samples, with the best-performing GAN model (SNGAN) under two settings. First, in Figures 5a and 5b, we show the influence of training the classifier with a combination of real images from the training set and $50 \mathrm{k}$ 


\begin{tabular}{l|c|c|c|c} 
Num real images & real C10 & real+GAN C10 & real C100 & real+GAN C100 \\
\hline $2.5 \mathrm{k}$ & 73.4 & 67.0 & 25.6 & 23.9 \\
$5 \mathrm{k}$ & 80.9 & 77.9 & 40.0 & 33.5 \\
$10 \mathrm{k}$ & 85.8 & 83.5 & 51.5 & 45.5
\end{tabular}

Table 4: Data augmentation when SNGAN is trained with reduced real image set. Classifier is trained either on this data (real) or a combination of real and SNGAN generated images (real+GAN). Performance is shown as \% accuracy.

GAN-generated images on the CIFAR10 and CIFAR100 datasets respectively. In this case, SNGAN is trained with all the images from the original training set. From both the figures, we observe that adding $2.5 \mathrm{k}$ or $5 \mathrm{k}$ real images to the 50k GAN-generated images improves the accuracy over the corresponding real-only counterparts. However, adding 50k real images does not provide any noticeable improvement, and in fact, reduces the performance slightly in the case of CIFAR100 (Figure 5b). This is potentially due to the lack of image diversity.

This experiment provides another perspective on the diversity of the generated set, given that the generated images are produced by a GAN learned from the entire CIFAR10 (or CIFAR100) training dataset. For example, augmenting $2.5 \mathrm{k}$ real images with $50 \mathrm{k}$ generated ones results in a better test accuracy than the model trained only on $5 \mathrm{k}$ real images. Thus, we can conclude that the GAN model generates images that have more diversity than the $2.5 \mathrm{k}$ real ones. This is however, assuming that the generated images are as realistic as the original data. In practice, the generated images tend to be lacking on the realistic front, and are more diverse than the real ones. These observations are in agreement with those from Section 5.3, i.e., SNGAN generates images that are at least as diverse as $5 \mathrm{k}$ randomly sampled real images.

In the second setting, SNGAN is trained in a low-data regime. In contrast to the previous experiment, we train SNGAN on a reduced training set, and then train the classifier on a combination of this reduced set, and the same number of generated images. Results in Table 4 show that on both CIFAR10 and CIFAR100 (C10 and C100 respectively in the table), the behaviour is consistent with the whole dataset setting (50k images), i.e., accuracy drops slightly.

\section{Summary}

This paper presents steps towards addressing the challenging problem of evaluating and comparing images generated by GANs. To this end, we present new quantitative measures, GAN-train and GAN-test, which are motivated by precision and recall scores popularly used in the evaluation of discriminative models. We evaluate several recent GAN approaches as well as other popular generative models with these measures. Our extensive experimental analysis demonstrates that GAN-train and GAN-test not only highlight the difference in performance of these methods, but are also complementary to existing scores. 


\section{References}

1. https://github.com/igul222/improved_wgan_training

2. https://github.com/bioinf-jku/TTUR

3. https://github.com/tkarras/progressive_growing_of_gans

4. Source code. http://thoth.inrialpes.fr/research/ganeval

5. Supplementary material, also available in arXiv technical report at https:// arxiv.org/abs/1807.09499

6. Arjovsky, M., Bottou, L.: Towards principled methods for training generative adversarial networks. In: ICLR (2017)

7. Arjovsky, M., Chintala, S., Bottou, L.: Wasserstein generative adversarial networks. In: ICML (2017)

8. Barratt, S., Sharma, R.: A note on the Inception score. arXiv preprint arXiv:1801.01973 (2018)

9. Berthelot, D., Schumm, T., Metz, L.: BEGAN: Boundary equilibrium generative adversarial networks. arXiv preprint arXiv:1703.10717 (2017)

10. Bousmalis, K., Silberman, N., Dohan, D., Erhan, D., Krishnan, D.: Unsupervised pixel-level domain adaptation with generative adversarial networks. In: CVPR (2017)

11. Calimeri, F., Marzullo, A., Stamile, C., Terracina, G.: Biomedical data augmentation using generative adversarial neural networks. In: ICANN (2017)

12. Chen, X., Duan, Y., Houthooft, R., Schulman, J., Sutskever, I., Abbeel, P.: InfoGAN: Interpretable representation learning by information maximizing generative adversarial nets. In: NIPS (2016)

13. Dai, Z., Yang, Z., Yang, F., Cohen, W.W., Salakhutdinov, R.R.: Good semisupervised learning that requires a bad GAN. In: NIPS (2017)

14. Deng, J., Dong, W., Socher, R., Li, L.J., Li, K., Fei-Fei, L.: ImageNet: A large-scale hierarchical image database. In: CVPR (2009)

15. Denton, E.L., Chintala, S., Szlam, A., Fergus, R.: Deep generative image models using a laplacian pyramid of adversarial networks. In: NIPS (2015)

16. Dumoulin, V., Belghazi, I., Poole, B., Mastropietro, O., Lamb, A., Arjovsky, M., Courville, A.: Adversarially learned inference. In: ICLR (2017)

17. Dumoulin, V., Shlens, J., Kudlur, M.: A learned representation for artistic style. In: ICLR (2017)

18. Frid-Adar, M., Klang, E., Amitai, M., Goldberger, J., Greenspan, H.: Synthetic data augmentation using GAN for improved liver lesion classification. In: ISBI (2018)

19. Goodfellow, I., Pouget-Abadie, J., Mirza, M., Xu, B., Warde-Farley, D., Ozair, S., Courville, A., Bengio, Y.: Generative adversarial nets. In: NIPS (2014)

20. Gulrajani, I., Ahmed, F., Arjovsky, M., Dumoulin, V., Courville, A.C.: Improved training of Wasserstein GANs. In: NIPS (2017)

21. He, K., Zhang, X., Ren, S., Sun, J.: Identity mappings in deep residual networks. In: $\operatorname{ECCV~(2016)~}$

22. Heusel, M., Ramsauer, H., Unterthiner, T., Nessler, B., Hochreiter, S.: GANs trained by a two time-scale update rule converge to a local Nash equilibrium. In: NIPS (2017)

23. Ho, T.K.: Random decision forests. In: ICDAR (1995)

24. Isola, P., Zhu, J.Y., Zhou, T., Efros, A.A.: Image-to-image translation with conditional adversarial networks. In: CVPR (2017) 
25. Karras, T., Aila, T., Laine, S., Lehtinen, J.: Progressive growing of GANs for improved quality, stability, and variation. In: ICLR (2018)

26. Kingma, D., Ba, J.: Adam: A method for stochastic optimization. In: ICLR (2015)

27. Kingma, D.P., Welling, M.: Auto-encoding variational Bayes. In: ICLR (2014)

28. Krizhevsky, A.: Learning multiple layers of features from tiny images. Tech. rep., University of Toronto (2009)

29. Krizhevsky, A., Sutskever, I., Hinton, G.E.: ImageNet classification with deep convolutional neural networks. In: NIPS (2012)

30. LeCun, Y., Bottou, L., Bengio, Y., Haffner, P.: Gradient-based learning applied to document recognition. Proc. IEEE 86(11), 2278-2324 (1998)

31. Ledig, C., Theis, L., Huszár, F., Caballero, J., Cunningham, A., Acosta, A., Aitken, A., Tejani, A., Totz, J., Wang, Z., et al.: Photo-realistic single image superresolution using a generative adversarial network. In: CVPR (2017)

32. Lucic, M., Kurach, K., Michalski, M., Gelly, S., Bousquet, O.: Are GANs created equal? A large-scale study. arXiv preprint arXiv:1711.10337 (2017)

33. van der Maaten, L., Hinton, G.: Visualizing data using t-SNE. JMLR 9(Nov), 2579-2605 (2008)

34. Mao, X., Li, Q., Xie, H., Lau, R.Y., Wang, Z., Smolley, S.P.: Least squares generative adversarial networks. In: ICCV (2017)

35. Mirza, M., Osindero, S.: Conditional generative adversarial nets. arXiv preprint arXiv:1411.1784 (2014)

36. Miyato, T., Kataoka, T., Koyama, M., Yoshida, Y.: Spectral normalization for generative adversarial networks. In: ICLR (2018)

37. Miyato, T., Koyama, M.: cGANs with projection discriminator. In: ICLR (2018)

38. Molano-Mazon, M., Onken, A., Piasini, E., Panzeri, S.: Synthesizing realistic neural population activity patterns using generative adversarial networks. In: ICLR (2018)

39. Mosser, L., Dubrule, O., Blunt, M.J.: Reconstruction of three-dimensional porous media using generative adversarial neural networks. Physical Review E 96(4), 043309 (2017)

40. Nowozin, S., Cseke, B., Tomioka, R.: f-GAN: Training generative neural samplers using variational divergence minimization. In: NIPS (2016)

41. Odena, A., Olah, C., Shlens, J.: Conditional image synthesis with auxiliary classifier GANs. In: ICML (2017)

42. Paganini, M., de Oliveira, L., Nachman, B.: Accelerating science with generative adversarial networks: An application to 3D particle showers in multilayer calorimeters. Physical review letters 120(4), 042003 (2018)

43. Pedregosa, F., Varoquaux, G., Gramfort, A., Michel, V., Thirion, B., Grisel, O., Blondel, M., Prettenhofer, P., Weiss, R., Dubourg, V., Vanderplas, J., Passos, A., Cournapeau, D., Brucher, M., Perrot, M., Duchesnay, E.: Scikit-learn: Machine learning in Python. JMLR 12, 2825-2830 (2011)

44. Rabin, J., Peyré, G., Delon, J., Bernot, M.: Wasserstein barycenter and its application to texture mixing. In: Intl. Conf. Scale Space and Variational Methods in Computer Vision (2011)

45. Radford, A., Metz, L., Chintala, S.: Unsupervised representation learning with deep convolutional generative adversarial networks. In: ICLR (2016)

46. Salimans, T., Goodfellow, I., Zaremba, W., Cheung, V., Radford, A., Chen, X.: Improved techniques for training GANs. In: NIPS (2016)

47. Salimans, T., Karpathy, A., Chen, X., Kingma, D.P.: PixelCNN++: Improving the PixelCNN with discretized logistic mixture likelihood and other modifications. In: ICLR (2017) 
48. Shin, H., Lee, J.K., Kim, J., Kim, J.: Continual learning with deep generative replay. In: NIPS (2017)

49. Shrivastava, A., Pfister, T., Tuzel, O., Susskind, J., Wang, W., Webb, R.: Learning from simulated and unsupervised images through adversarial training. In: CVPR (2017)

50. Szegedy, C., Liu, W., Jia, Y., Sermanet, P., Reed, S., Anguelov, D., Erhan, D., Vanhoucke, V., Rabinovich, A., et al.: Going deeper with convolutions. In: CVPR (2015)

51. Theis, L., Oord, A.v.d., Bethge, M.: A note on the evaluation of generative models. In: $\operatorname{ICLR}(2016)$

52. Tran, T., Pham, T., Carneiro, G., Palmer, L., Reid, I.: A Bayesian data augmentation approach for learning deep models. In: NIPS (2017)

53. Van Den Oord, A., Kalchbrenner, N., Kavukcuoglu, K.: Pixel recurrent neural networks. In: ICML (2016)

54. Wang, Y.X., Girshick, R., Hebert, M., Hariharan, B.: Low-shot learning from imaginary data. In: CVPR (2018)

55. Yun, K., Bustos, J., Lu, T.: Predicting rapid fire growth (flashover) using conditional generative adversarial networks. arXiv preprint arXiv:1801.09804 (2018)

56. Zhang, H., Xu, T., Li, H., Zhang, S., Huang, X., Wang, X., Metaxas, D.: StackGAN: Text to photo-realistic image synthesis with stacked generative adversarial networks. In: ICCV (2017)

57. Zhao, J., Mathieu, M., LeCun, Y.: Energy-based generative adversarial networks. In: ICLR (2017)

58. Zhong, Z., Zheng, L., Zheng, Z., Li, S., Yang, Y.: Camera style adaptation for person re-identification. In: CVPR (2018)

59. Zhu, J.Y., Park, T., Isola, P., Efros, A.A.: Unpaired image-to-image translation using cycle-consistent adversarial networks. In: ICCV (2017) 


\section{Appendix A: Implementation details}

SNGAN in our experiments refers to the model with ResNet architecture, hinge loss, spectral normalization [36], conditional batch normalization [17] and conditioning via projection [37]. We evaluate two variants of WGAN-GP: one with $2.5 \mathrm{M}$ and the other with $10 \mathrm{M}$ parameters. Both these variants use ResNet architecture from [20], Wasserstein loss [7] with gradient penalty [20] and conditioning via an auxiliary classifier [41], with the only difference being that, there are twice as many filters in each layer of the generator for the $10 \mathrm{M}$ model over the $2.5 \mathrm{M}$ variant. We use DCGAN with a simple convnet architecture [45], classical GAN loss, conditioned via an auxiliary classifier [41], as in [36].

We reimplemented WGAN-GP, SNGAN, DCGAN, and validated our implementations on CIFAR10 to ensure that they match the published results. Our implementations are available online [4]. For PixelCNN++, we used the reference implementation $^{1}$ for training and the accelerated version ${ }^{2}$ for inference.

For all the CIFAR experiments, SNGAN and WGAN-GP are trained for 100k iterations with a linearly decaying learning rate, starting from 0.0002 to 0 , using the Adam optimizer [26] $\left(\beta_{1}=0, \beta_{2}=0.9,5\right.$ critic steps per generator step, and generator batch size 64). ACGAN loss scaling coefficients are 1 and 0.1 for the critic's and generator's ACGAN losses respectively. Gradient penalty is 10 as recommended [20]. DCGAN is also trained for 100k iterations with learning rate 0.0002 , Adam parameters $\beta_{1}=0.5, \beta_{2}=0.999$, and batch size of 100 .

In the case of ImageNet, we follow [37] for ResNet architecture and the protocol. We trained for $250 \mathrm{k}$ iterations with learning rate 0.0002 , linearly decaying to 0 , starting from the $200 \mathrm{k}$-th iteration, for resolution $64 \times 64$. We use the Adam optimizer [26] $\left(\beta_{1}=0, \beta_{2}=0.9,5\right.$ critic steps per generator step, generator batch size 64 ). For $128 \times 128$ resolution, we train for $450 \mathrm{k}$ iterations, with learning rate linearly decaying to 0 , starting from the $400 \mathrm{k}$-th iteration. WGANGP for ImageNet uses the same ResNet and training schedule as SNGAN. Its gradient penalty and ACGAN loss coefficients are identical to the CIFAR case.

The classifier for computing GAN-train and GAN-test is a preactivation variant of ResNet-32 from [21] in the CIFAR10 and CIFAR100 evaluation. Training schedule is identical to the original paper: $64 \mathrm{k}$ iterations using momentum optimizer with learning rate 0.1 dropped to 0.01 , after $32 \mathrm{k}$ iterations, and 0.001 , after $48 \mathrm{k}$ iterations (batch size 128). We also use standard CIFAR data augmentation: $32 \times 32$ crops are randomly sampled from the padded image (4 pixels on each side), which are flipped horizontally. The classifier in the case of ImageNet is ResNet-32 for $64 \times 64$ and ResNet-34 for $128 \times 128$ with momentum optimizer for $400 \mathrm{k}$ iterations, and learning rate 0.1 , dropped to 0.01 after $200 \mathrm{k}$ steps (batch size 128). We use a central crop for both training and testing. We also compute our measures with a random forest classifier [23]. Here, we used the scikit-learn implementation [43] with 100 trees and no depth limitation.

\footnotetext{
${ }^{1}$ https://github.com/openai/pixel-cnn

${ }^{2}$ https://github.com/PrajitR/fast-pixel-cnn
} 
To train SNGAN on MNIST we use the same GAN architecture as in the case of CIFAR10 (adjusted to a single input channel), and a simple convnet architecture (four convolutional layers with 32, 64, 128, 256 filters with batch normalization and max pooling in between, and global average pooling before the final output layer) as the baseline classifier. The GAN training schedule is also the same as in the case of CIFAR 10 . We train the baseline classifier for $64 \mathrm{k}$ iterations using a momentum optimizer with learning rate 0.1 dropped to 0.01 , after $32 \mathrm{k}$ iterations, and 0.001 , after $48 \mathrm{k}$ iterations (using a batch size of 128).

\section{Appendix B: Size comparison}

To complete the discussion on dataset memorization, we compare the size of the generator and the real dataset. In our CIFAR experiments SNGAN has $8.3 \mathrm{M}$ parameters $(33 \mathrm{Mb})$, WGAN-GP has either $2.5 \mathrm{M}$ or $10 \mathrm{M}$ parameters $(10 \mathrm{Mb}$ or $40 \mathrm{Mb}$ respectively). In comparison, CIFAR $10 / 100$ have 50k training images which amounts to $150 \mathrm{Mb}$ of uncompressed data.

SNGAN for ImageNet has $42 \mathrm{M}$ parameters (168Mb) and WGAN-GP has $48 \mathrm{M}$ parameters $(192 \mathrm{Mb})$. The entire ImageNet training set takes $16 \mathrm{~Gb}$ in $64 \times 64$ resolution and $64 \mathrm{~Gb}$ in $128 \times 128$ resolution. This difference may partially explain why compression of CIFAR10/100 into a GAN is relatively easier than ImageNet.

\section{Appendix C: t-SNE Embeddings of Datasets}

In Figure 6 we show t-SNE embedding of randomly selected images from the CIFAR100 train and test set splits, and images generated from the four GAN models (500 images per split or model) of 5 classes (apple, aquarium fish, baby, bear, bicycle). The t-SNE visualizations are generated with the embeddings of these images in the feature space of the baseline classifier trained on CIFAR100, i.e., the classifier used to compute GAN-test accuracy. Note that the quality of this clustering is in line with the GAN-test accuracy in Table 2 in the main paper. For example, the images generated by SNGAN and WGAN-GP (10M), which produce the two best GAN-test accuracies, lie close to the training images, while those from WGAN-GP $(2.5 \mathrm{M})$ and DCGAN form a point cloud that does not correspond to any of the clusters. 

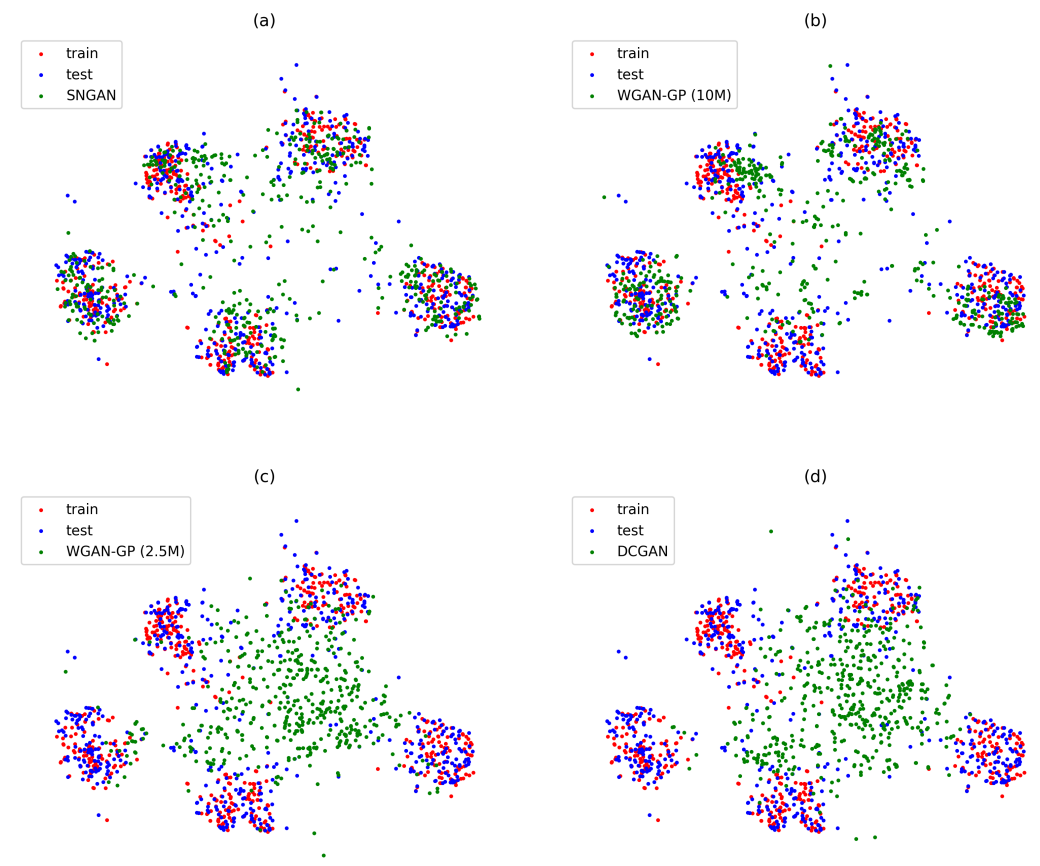

Fig. 6: t-SNE [33] embedding of images from 5 CIFAR100 classes, embedded in the feature space of the baseline CIFAR100 classifier. We use 500 images each from the train and test sets, along with 500 images each generated with (a) SNGAN, (b) WGAN-GP (10M), (c) WGAN-GP (2.5M), and (d) DCGAN. 